\title{
Infections of the spine are still a great problem
}

\author{
Luis Lopez-Duran Stern • Carlos Leon Serrano
}

Published online: 9 February 2012

(C) Springer-Verlag 2012

Infections of the spine are still a great problem in many countries, which is the reason the editorial board asked us to devote this special annual issue to the subject. We have arranged the content into different sections to present an idea of the incidence, diagnostic problems and surgical treatments regarding spinal infections.

Tuberculosis (TB) is a very old disease and is the most frequent cause of spinal infections. Its incidence has increased since 1980, making it responsible for more than two million deaths worldwide [1]. Extrapulmonary TB accounts about $15-20 \%$ of TB cases, and although the true incidence of spinal TB is not known, it is estimated to occur in $1-5 \%$ of all TB cases. In data collected by Merino et al., the incidence of Pott's disease is in the order of 0.7-2.4 cases per 100,000 inhabitants per year in Spain [3]. The clinical study by Kamara et al. [4] shows the detrimental effect of a delay in diagnosis, which in their geographical area was a median of five months and varied with patient age, education level and religion.

Diagnosis is based mostly on clinical data, and is neither always easy nor easily confirmed bacteriologically, as evidenced in $59.3 \%$ and $45.2 \%$ positive Ziehl-Neelsen stain in the series by Merino et al. [2]. Introduction of the polymerase chain reaction (PCR) technique allows a more accurate diagnosis. Sharma et al. reports a study of two target genes that were found to be specific for Mycobacterium TB in 80 patients; multiple PCR had a sensitivity of $81.8 \%$ in

L. Lopez-Duran Stern $(\bowtie) \cdot$ C. Leon Serrano Departement of Trauma and Orthopaedic Surgery, Hospital Clínico San Carlos, Universidad Complutense, Madrid, Spain

e-mail: 1lopezduran@hotmail.com suspected cases of peripheral TB osteoarthritis [5]. This promising test for TB diagnosis has the advantage of being fast; however, it is expensive and it is not available everywhere. Jain et al. [6] report the value of magnetic resonance (MR) as a diagnostic tool for Pott's disease and compare the value of X-rays, with a sensitivity, specificity and accuracy of $82 \%, 57 \%$ and $73 \%$, respectively, for $\mathrm{MR}$ findings of marrow oedema $(100 \%)$, pre- and paravertebral septate loculated collections (100\%), subligamentous collections (92\%) and endplate erosions (99\%) in the cases studied. Preservation of discs despite extensive bone destruction is virtually pathognomonic for spinal TB. MR images in surgically treated cases are often confusing due to the metallic implants, and there is a search for other diagnostic tools such as the technetium-99m ciprofloxacin scan to assess disease activity. In a prospective study, Agrawal et al. [7] found a good correlation between the clinicoradiological resolution after six months of treatment and scan normalisation .

Controversy about the duration of the medical treatment of Pott's disease continues, although it is not the subject of this special issue. Nevertheless, poorly monitored treatment or its early withdrawal seems to increase the number of drug-resistant cases, as reported by Li et al., with $30.7 \%$ of drug-resistant cases in their series, and presents a study and treatment protocol for them [8]. The indications and surgical technique for treating spinal TB offer some different approaches, which we present in a section beginning with the revision article of Jain and Jain [9]. Zhang et al. recommend a posterior approach only [10] for thoracic monosegmental lesions and compare this technique of debridement by the posterior approach associated with posterior fixation with an anterior and posterior double approach. There were no differences in kyphosis correction or bone consolidation, 
but the posterior only method was easier. The articles by Ma et al., Pu et al., Wang et al. and El-Sharkawi et al. [11-14] compare the combination of an anterior and posterior approach in different protocols. Ma et al. [11] report a better deformity correction using posterior instrumentation. Pu et al. [12] show the same results with the anterior and posterior techniques and suggest that the posterior approach with focus debridement is adequate. Wang et al. [13] recommend the monosegmental fixation for cases with destruction between one third and two thirds of body height. El-Sharkawi et al. [14] found similar results by comparing a circumferential fusion through a posterior approach with a staged anterior and posterior intervention, but they recommended the posterior approach only due to the lower complication rate, shorter hospital stay and less operative time and blood loss.

The use of thoracoscopy to treat anterior lesions of the thoracic spine may be indicated in cases with less than $50 \%$ vertebral body destruction and a kyphotic angle under $30^{\circ}$, cases in which the aim is to debride the focus and, if necessary, add a rib graft. With this technique, Kapoor et al. report very promising results [15]. Controversies about the best surgical technique to manage cases in the lumbosacral level persist $[16,17]$. With the anterior approach, radical debridement, autologous grafting and instrumentation, Song et al. [16] reported good results. They compared both methods, anterior only or the combination of both approaches with good results in each, but they recommend using the anterior approach as the first choice.

A possible conclusion that can be drawn from this section is that surgical treatment should combine vertebral body debridement using a thoracoscopic, anterior or posterior approach, with filling of the destroyed area using grafts and/or cages and a stable fixation, which seems to be easier to accomplish by posterior instrumentation; also, it seems that the use of metallic implants does not increase the risk of a persistent TB infection, a secondary bacterial infection or a delay in healing and consolidation of vertebral lesions.

The next section is dedicated to correcting the residual kyphotic deformities of the Pott's spine, and begins with the review article by Issack and Boachie-Adjei [18], who emphasise that progressive neurological deficit is the worst complication of kyphotic deformity and consider the different surgical options and their risks. In his review article, Rajasekaran [19] describes the buckling collapse, the spineat-risk signs and surgery for active disease or established deformity. Liu et al. [20] present a series of cervical kyphosis treated through an anterior approach, with debridement and reconstruction using grafts or cages accomplishing a correction in the order of $50^{\circ}$. Wang et al. [21] review a group of thoracic and lumbar cases treated by instrumented posterior fusion combined with an anterior procedure, resulting in a correction of $40^{\circ}$ and an improvement of 1.5 grades in the Frankel scale. The surgical results of Zhang
[22] for treating cases of late-onset paraplegia with severe kyphosis had a poor prognosis after surgery.

After the articles devoted to Pott's disease, there is a group of reports dedicated to spondylodiscitis. De Moraes Barros Fucs et al. [23] present a review article focused on children's spondylodiscitis, a rare disease of unknown incidence. Cheung and Luk, Zarghooni et al. and Guerado and Cervan [24-26] each wrote a review article about the incidence and treatment of spondylodiscitis in adults, Cheung and Luk [24] describe in detail the clinical and diagnostic protocols to follow in these cases, Zarghooni et al. [25] present a treatment algorithm and Guerado and Cervan [26] discusses surgical controversies. Cervan et al. [27] collected an important number of spondylodiscitis in patients on haemodialysis. The diagnosis is frequently made very late; MR is the best way to diagnose and localise the focus in this serious complication in dialysis patients, for whom the authors recommend prompt surgery. There are other specific types of spinal infection, such as those due to Salmonella and Brucella. Less frequent but much more interesting are the hydatid spinal infections that can be compared with malignant tumours in respect to mortality, aggressive behaviour and the impossibility of total eradication in most cases, as described in the article by Hamdan [28].

The final section of this issue is devoted to postoperative spinal infections. Meredith et al. [29] describe in their review the different risk factors and modes of presentation. Hamdan [30] analyses a series of 35 cases of disc-space infections, their clinical presentation and treatment results. Quaile, and Gerometta et al. [31, 32] present review articles about infections associated with spinal implants. Quaile [31] discusses the value of the prophylactic antibiotic treatment and the need for early diagnosis and treatment in these infections. Gerometta et al. [32] focus on the controversy over retention versus removal of instrumentation. Bachy et al. [33] review infections after spinal correction and fusion, presenting an algorithm of treatment and stressing the risk of progressive spinal deformity after implant removal in late infections. The last article [34] is a review of the risks and possibilities of treatment in the case of a total disc arthroplasty infection.

\section{References}

1. Fuentes M, Gutiérrez L, Ayala O, Rumayor M, Prado N (2012) Tuberculosis of the spine. A systematic review of case series. Int Orthop 1:2. doi:10.1007/s00264-011-1414-4

2. Merino P, Candel F, Gestoso I, Baos E, Picazo J (2012) Microbiological diagnosis of spinal tuberculosis. Int Orthop. doi:10.1007/ s00264-011-1461-x

3. Cebrián JL, Saez-Arenillas A, Urda A, Soler I, Agreda E, LopezDuran L (2012) Management of infectious discitis. Outcome in one hundred and eight patients in a University Hospital. Int Orthop 1:2. doi:10.1007/s00264-011-1445-x 
4. Kamara E, Mehta S, Brust J, Jain A (2012) Effect of delayed diagnosis on severity of Pott's disease. Int Orthop 1:2. doi:10.1007/s00264-011-1432-2

5. Sharma K, Sharma A, Sharma SK, Kumar Sen R, Dhillon MS, Sharma M (2012) Does multiplex Polymerase Chain Reaction increase the diagnostic percentage in osteoarticular tuberculosis? A prospective evaluation of 80 cases. Int Orthop 1:2. doi:10.1007/ s00264-011-1241-7

6. Jain A, Sreenivasan R, Saini R, Kumar S, Jain S, Dhammi K (2012) Magnetic Resonance evaluation of tubercular lesion in spine. Int Orthop 1:2. doi:10.1007/s00264-011-1380-x

7. Agrawal M, Bhardwaj V, Tsering W, Sural S, Kashyap R, Dahl A (2012) Use of Technetium ${ }^{99 m}$-ciprofloxacin scan in Pott's spine to assess the disease activity. Int Orthop 1:2. doi:10.1007/s00264011-1310-y

8. Li L, Zhang Z, Luo F, Xu J, Cheng P, Wu Z, Zhou O, He O et al (2012) Management of drug-resistant spinal tuberculosis with a combination of surgery and individualised chemotherapy: a retrospective analysis of thirty-five patients. Int Orthop 1:2. doi:10.1007/s00264-011-1398-0

9. Jain A, Jain S (2012) Instrumented stabilization in spinal tuberculosis. Int Orthop 1:2. doi:10.1007/s00264-011-1296-5

10. Zhang H, Huang S, Guo H, Ge L, Sheng B, Wang Y et al (2012) A clinical study of internal fixation, debridement and interbody thoracic fusion to treat thoracic tuberculosis via posterior approach only. Int Orthop 1:2. doi:10.1007/s00264-011-1449-6

11. Ma YZ, Cui X, Li H, Chen X, Cai X, Bai Y (2012) Outcomes of anterior and posterior instrumentation under different surgical procedures for treating thoracic and lumbar spinal tuberculosis in adults. Int Orthop 1:2. doi:10.1007/s00264-011-1390-8

12. Pu X, Zhou O, He O, Dai F, Xu J, Zhang Z, Branko K (2012) A posterior versus anterior surgical approach in combination with debridement, interbody autografting and instrumentation for thoracic and lumbar tuberculosis. Int Orthop 1:2. doi:10.1007/s00264011-1329-0

13. El-Sharkawi MM, Said GK (2012) Instrumented circumferential fusion for tuberculosis of the dorso-lumbar spine. A single or double stage procedure? Int Orthop 1:2. doi:10.1007/s00264-011-1401-9

14. Wang Z, Yuan H, Geng G, Shi J, Jin W (2012) Posterior monosegmental fixation, combined with anterior debridement and strut graft, for treatment of the mono-segmental lumbar spine tuberculosis. Int Orthop 1:2. doi:10.1007/s00264-011-1475-4

15. Kapoor S, Kapoor S, Agrawal M, Aggarwal P, Jain BK (2012) Thoracoscopic decompression in Pott's spine and its long-term follow-up. Int Orthop 1:2. doi:10.1007/s00264-011-1453-x

16. Song J-F, Jing Z-Z, Chen B, Ai Z-S, Wei H (2012) One-stage anterolateral surgical treatment for lumbosacral segment tuberculosis. Int Orthop 1:2. doi:10.1007/s00264-011-1378-4
17. He Q, Jianzhong Xu (2012) Comparison between the anteroposterior and anterior approaches for treating L5-S1 vertebral tuberculosis. Int Orthop 1:2. doi:10.1007/s00264-011-1307-6

18. Issack P, Boachie-Adjei O (2012) Surgical correction of kyphotic deformity in spinal tuberculosis. doi:10.1007/s00264-011-1292-9

19. Rajasekaran S (2012) Kyphotic deformity in spinal tuberculosis and its management. Int Orthop 1:2. doi:10.1007/s00264-011-1469-2

20. Liu Y, Chen Y, Zhou X, Wang C, Qi M, Yuan W (2012) The surgical treatment and related management for post-tubercular kyphotic deformity of the cervical spine or the cervico-thoracic spine. Int Orthop 1:2. doi:10.1007/s00264-011-1438-9

21. Wang X, Li J, Lü G, Wang B, Lu Ch, Kang YY (2012) Singlestage posterior instrumentation and anterior debridement for active tuberculosis of the thoracic and lumbar spine with kyphotic deformity. Int Orthop 1:2. doi:10.1007/s00264-011-1389-1

22. Zhang Z (2012) Late onset Pott's paraplegia in patients with upper thoracic sharp kyphosis. Int Orthop 1:2. doi:10.1007/s00264-0111285-8

23. Fucs P, Meves R, Yamada HH (2012) Spinal infections in children: a review. Int Orthop 1:2. doi:10.1007/s00264-011-1388-2

24. Cheung WY, Luk K (2012) Pyogenic spondylitis. Int Orthop 1:2. doi:10.1007/s00264-011-1384-6

25. Zarghooni K, Röllinghoff M, Sobottke R, Eysel P (2012) Treatment of spondylodiscitis. Int Orthop 1:2. doi:10.1007/s00264-011-1425-1

26. Guerado E, Cervan AM (2012) Surgical treatment of spondylodiscitis. An update. Int Orthop 1:2. doi:10.1007/s00264-011-1441-1

27. Cervan AM, Colmenero J, Arco A, Villanueva F, Guerado E (2012) Spondylodiscitis in patients under haemodyalisis. Int Orthop 1:2. doi:10.1007/s00264-011-1433-1

28. Hamdan T (2012) Hydatid disease of the spine: a report on 9 patients. Int Orthop 1:2. doi:10.1007/s00264-011-1480-7

29. Meredith D, Kepler Ch, Huang R, Brause B, Boachie-Adjei O (2012) Postoperative infections of the lumbar spine: presentation and management. Int Orthop 1:2. doi:10.1007/s00264-011-1427-z

30. Hamdan T (2012) Postoperative disc space infection after discectomy: a report on thirty-five patients. Int Orthop. doi:10.1007/ s00264-011-1430-4

31. Quaile A (2012) Infections associated with spinal implants: a review article. Int Orthop 1:2. doi:10.1007/s00264-011-1408-2

32. Gerometta A, Rodriguez Olaverri JC, Bitan F (2012) Infections in spinal instrumentation. Int Orthop 1:2. doi:10.1007/s00264-0111426-0

33. Bachy M, Bouyer B, Vialle R (2012) Infections after spinal correction and fusion for spinal deformities in childhood and adolescence. Int Orthop 1:2. doi:10.1007/s00264-011-1439-8

34. Gerometta A, Rodriguez Olaverri JC, Bittan F (2012) Infection and revision strategies in total disc arthroplasty. Int Orthop 1:2. doi:10.1007/s00264-011-1436-y 